\title{
TranCEP: Predicting transmembrane transport proteins using composition, evolutionary, and positional information
}

\author{
Munira Alballa ${ }^{1}$, Faizah Aplop², Gregory Butler ${ }^{1,3^{*}}$
}

1 Department of Computer Science and Software Engineering, Concordia University, Montréal, Québec, Canada

2 School of Informatics and Applied Mathematics, Universiti Malaysia Terengganu, Malaysia

3 Centre for Structural and Functional Genomics, Concordia University, Montréal, Québec, Canada

* gregory.butler@concordia.ca

\begin{abstract}
Transporters mediate the movement of compounds across the membranes that separate the cell from its environment, and across inner membranes surrounding cellular compartments. It is estimated that one third of a proteome consists of membrane proteins, and many of these are transport proteins. Given the increase in the number of genomes being sequenced, there is a need for computation tools that predict the substrates which are transported by the transmembrane transport proteins.

In this paper, we present TranCEP, a predictor of the type of substrate transported by a transmembrane transport protein. TranCEP combines the traditional use of the amino acid composition of the protein, with evolutionary information captured in a multiple sequence alignment, and restriction to important positions of the alignment that play a role in determining specificity of the protein.

Our experimental results show that TranCEP significantly outperforms the state of the art. The results quantify the contribution made by each kind of information used.

Transmembrane proteins are gates that organize a variety of vital cellular functions including cell signaling, trafficking, metabolism, and energy production. It is estimated that on average one in every three proteins in a cell is a membrane protein [Bue15, KST13. Any defective or misregulated membrane protein can disturb the organism's functioning, giving rise to disease [GO14. About one-half of the drug targets today are membrane proteins such as transporters or related receptors [BRK17]. While the amino acid sequence of many membrane proteins is available, they are among the least characterized proteins in terms of their structure and function. For example, only $3 \%$ of the structures in the PDB are transmembrane proteins.
\end{abstract}

\section{Introduction}


The publication of numerous genome projects has produced an abundance of protein sequences, many of which remain uncharacterized; transmembrane proteins are among the least characterized proteins, because experimental characterization of their structure and function is exceptionally difficult, owing to their hydrophobic surfaces and their lack of conformational stability. Consequently, there is an urgent need for computational approaches that use the available data to distinguish and characterize transmembrane proteins. Yet, this area of research is still in its early stages, and the researchers are far from finding a definitive solution.

Existing tools for the annotation of transporters that predict the substrate for the transport reaction lag behind tools for other kinds of proteins such as enzymes for metabolic reactions. Most tools predict the type of substrates [SCH10, COLG11, SH12, BH13, MCZ14], chosen from a small subset of substrate types, without attempting to predict the specific substrate, or predict the family or subfamily [GY08, LBUZ09, OCG10, BH13] for the protein within the Transporter Classification (TC) [BSJ03, SJTB06, SJRT ${ }^{+}$16], again without attempting to predict the specific substrate. For network modeling in systems biology [TP10, SAJT14, we require tools to process the complete proteome and predict each transport reaction; this means identifying the transport protein and the specific substrate.

Many tools rely simply on homology or orthology to predict transporters. This includes metabolic network tools merlin [DRFR15], and Pantograph [LZS15], as well as our system TransATH [AB17]. Amongst the tools for de novo prediction of substrate class, TrSSP [MCZ14] claims to be the state of the art.

Our previous efforts Apl16 for de novo prediction of specific substrates for sugar transporters in fungi were not successful. However, from it we learned how much depends on very few residues of the transporter; often three or so residues, and often internal to different helix transmembrane segments (TMS) of the transporter $\left[\mathrm{FBS}^{+} 14\right]$. These residues are far apart in the linear protein sequence, but are close to each in the three-dimensional structure of the protein when integrated in the membrane. In looking forward to how we might improve on approaches that rely on the amino acid composition of the protein, we developed a roadmap where the composition information would be combined with evolutionary information as captured by a multiple sequence alignment (MSA), and by positional information [TWNB12] about the residues responsible for determining specificity of the transporter. This roadmap is a schema for a large number of possible algorithms due to the many choices for encoding of amino acid composition, MSA algorithms, and algorithms for specificity determining sites [CC14]. We also realized the importance of the alignment preserving the TMS positions since the important residue positions seem to be located there. There are a number of such MSA algorithms [PFH08, CDTTN12, $\left.\mathrm{FTC}^{+} 16, \mathrm{BGA}^{+} 17\right]$.

Here, we conduct a preliminary study that shows the combination of information about protein composition, protein evolution, and the specificity determining positions has significant impact on our ability to predict the transported substrates. We chose the methodology and datasets of TrSSP [MCZ14] as our baseline, and varied this to illustrate the impact of each of the factors: compositional, evolutionary, and positional information. Our best approach, which defines the predictor we call TranCEP, for Transporter prediction using Compositional, Evolutionary, and Positional information, adopts the PAAC encoding scheme, the TM-Coffee MSA algorithm, and the TCS algorithm for determining informative positions in the MSA, to build a suite of Support Vector Machine (SVM) classifiers, one for distinguishing between each pair of classes of substrates. 


\section{$1.1 \quad$ Background}

For most of the work done on the prediction of transport proteins [GO14], there is no available software, so it is difficult to reproduce the work and to compare the results of different articles.

TransAAP [RKP04] is a semi-automated analysis pipeline to input data into TransportDB. TransAAP targets only prokaryotes. A new genome is matched against the curated set of TransportDB proteins with assigned family using Blast with e-value cut-off of 1e-3. Information from these Blast searches against TransportDB are collected, as is information from searches against non-transporters in the nr protein database, and classification by orthology using COG. A web-based interface displays the information to help a human annotator decide and assign possible substrates or functions.

Pathway Tools includes the Transport Inference Parser (TIP) [LPK08] which analyses keywords in a gene annotation to assign Gene-Protein-Reaction associations to transport reactions in MetaCyc. G-Blast [RS12] screens proteins against all entries in TCDB using Blast to retrieve the top hit, and HMMTOP to determine information about TMSs for the query and the hit sequence. It is an integral part of a manual protocol of Saier's lab to predict the transport proteins for a genome [PVL $\left.{ }^{+} 14\right]$.

The Zhao Lab has developed three methods: a nearest neighbour approach [LDZ08]; TransportTP [LBUZ09]; and TrSSP [MCZ14]. The nearest neighbour approach achieved a balanced accuracy of $67.0 \%$.

TransportTP [LBUZ09] is a two-phase algorithm that combines homology and machine learning to predict TC family of one or more proteins. For training and cross-validation testing, TransportTP used the yeast proteome. For testing, it used 10 genomes from the TransportDB database [RCP07] of annotated prokaryote transporters. As an independent test, TransportTP is trained on the proteome of $A$. thaliana and then used to predict the transporters in four other plant proteomes. TransportTP achieved a balanced accuracy of $81.8 \%$. Compared with the SVM-Prot classifier [ $\mathrm{LHC}^{+} 06$ ], on the five TC superfamilies and three families used by SVM-Prot, TransportTP achieved better recall and precision.

TrSSP (Transporter Substrate Specificity Prediction Server) [MCZ14 is a web server to predict membrane transport proteins and their substrate category. The substrate categories are: (1) oligopeptides (amino acid); (2) anion; (3) cation; (4) electron; (5) protein/mRNA; (6) sugar; and (7) other. TrSSP makes a top-level prediction of whether the protein is a transporter, or not. A SVM is applied with highest accuracy being reported using Amino Acid index (AAindex) and Position-Specific Scoring Matrix (PSSM).

The disc_function system [GY08] uses amino acid composition and neural networks for discriminating channels/pores, electrochemical and active transporters, with an accuracy of $68 \%$. When augmented with PSSM profiles and amino acid physicochemical properties they gained $5-10 \%$ in discrimination accuracy [OCG10]. They also considered six major families in TCDB [OCG10] with an average accuracy of $69 \%$.

$T T R B F$ COLG11 considers four major classes of substrates: (i) electron, (ii) protein/mRNA, (iii) ion and (iv) others. It is an ensemble system combining amino acid composition, dipeptide composition, physicochemical properties, PSSM profiles and radial basis function (RBF) networks.

Schaadt et al. SCH10] used amino acid composition, characteristics of amino acid residues and conservation to detect transporters based on different substrates, amino acids, oligopeptides, phosphates 
and hexoses and showed an accuracy of $75 \%$ to $90 \%$. They classified to four substrate categories: amino acid, oligopeptide, phosphate, and hexose. The number of characterized transporters in $A$. thaliana for the four substrates numbered from 13 to 17. They constructed a vector for each protein using various types of amino acid composition, AAC, PAAC, PseAAC, PsePAAC, MSA-AAC, and used Euclidean distance from the query protein's vector to the known vectors to rank the substrate categories. They found that AAC did not yield accurate results. However, PAAC performed as well as the more complicated PsePAAC and MSA-AAC, yielding accuracy over $90 \%$.

Schaadt and Helms [SH12] compared the similarity of transporters in TCDB and annotated transporters in $A$. thaliana using amino acid composition and classified the proteins into three families. By distinguishing the amino acid composition of TMS and non-TMS regions, they could classify four different families with an accuracy of $80 \%$.

Barghash and Helms [BH13] performed a comparison of three different approaches (homology, HMMER, MEME) for predicting substrate category and predicting TC family. They used four substrate categories, metal ions, phosphate, sugar, and amino acid; and $29 \mathrm{TC}$ families with the most numerous examples. The datasets are from E. coli, S. cerevisiae, and A. thaliana, consisting of the 155, 158, 177, respectively, proteins that had both a substrate annotation and TC family annotation that are experimentally determined.

Pantograph [LZS15] was designed [Loi12] for metabolic pathway reconstruction of yeasts such as Yarrowia lipolytica [LDNS12] which accumulates lipids in the peroxisome component of the cell. It specifically models the cellular components and the transport across the membranes in a reference template, called the scaffold model. The Pantograph method relies on a database of profile HMMs for fungal protein families and their annotations that is maintained at Génolevures in Bordeaux. The Pantograph algorithm first assigns Gene-Protein-Reaction associations, and then decides which compartments and reactions to include in the draft model based on these associations.

The scaffold model, which is the reference template for Pantograph, was manually curated to include 421 transport reactions. The associated transport protein families of orthologs were manually identified in the Génolevures collection. The Pantograph software, written in Python, is available at http://pathtastic.gforge.inria.fr/. The distribution includes the scaffold model in SBML (Systems Biology Markup Language).

The merlin [DRFR15] system for the reconstruction of metabolic networks handles eukaryote genomes, and includes the determination of transport Gene-Protein-Reaction associations, as well as localization of reactions across a number of compartments: mitochondrion, endoplasmic reticulum (ER), and Golgi apparatus. In merlin, transport proteins are predicted based on the existence of TMS as predicted by TMHMM, and by similarity to entries in TCDB using the Smith-Waterman algorithm. The association of transport reactions and specific substrates for the predicted transport proteins is taken from a manually curated database of some 4000 TCDB entries originally. It now incorporates TRIAGE (Transport Reactions Annotation and Generation) [DGV ${ }^{+} 17$ ] which contains information for 5,495 TCDB entries. The merlin software is available as open source Java code (http://www.merlin-sysbio.org).

TransATH [AB17] (Transporters via Annotation Transfer by Homology) is a system which automates Saier's protocol based on sequence similarity. TransATH includes the computation of subcellular localization and improves the computation of transmembrane segments. The parameters of TransATH 
are chosen for optimal performance on a gold standard set of transporters and non-transporters from S. cerevisiae. A website http://transath.umt.edu.my for TransATH is available for use.

SCMMTP [LVY ${ }^{+}$] $]$uses a novel scoring card method (SCM) that utilizes the use of dipeptide composition to identify putative membrane transport proteins. The SCMMTP method first builds an initial matrix of 400 dipeptides and uses the difference between compositions of positives and negatives as an initial dipeptide scoring matrix. This matrix is then optimized using a genetic algorithm. SCMMP achieved an overall accuracy of $76.11 \%$ and Matthews correlation coefficient (MCC) of 0.47 in the independent dataset.

Li et al $\left[\mathrm{LLX}^{+} 16\right]$ used a SVM to predict substrate classes of transmembrane transport proteins by integrating features from PSSM, amino acid composition, biochemical properties and Gene Ontology (GO) terms. They achieved an overall accuracy of $80.45 \%$ on the independent dataset.

\section{Materials and methods}

\section{$2.1 \quad$ Datasets}

We used the same training dataset and test dataset as TrSSP [MCZ14 available at http://bioinfo. noble.org/TrSSP taken from Swiss-Prot (release 2013-03). Table 1 shows the statistics for the seven substrate classes considered: amino acid, anion, cation, electron, protein/mRNA, sugar, and other. The class other refers to transporters that do not belong to any of the other six classes. The dataset consisted of 760 transporters and 120 for the test dataset.

\begin{tabular}{|c|c|c|}
\hline $\begin{array}{c}\text { Substrate } \\
\text { class }\end{array}$ & $\begin{array}{c}\text { Training } \\
\text { dataset }\end{array}$ & $\begin{array}{c}\text { Test } \\
\text { dataset }\end{array}$ \\
\hline Amino acid & 70 & 15 \\
\hline Anion & 60 & 12 \\
\hline Electron & 60 & 10 \\
\hline Cation & 260 & 36 \\
\hline Protein/mRNA & 70 & 15 \\
\hline Sugar & 60 & 12 \\
\hline Other & 200 & 20 \\
\hline \hline Total & 780 & 120 \\
\hline
\end{tabular}

Table 1. The dataset.

\subsection{Databases}

We use the Swiss-Prot database when searching for similar sequences. TM-Coffee uses the UniRef50-TM database, which consists of the entries in UniRef50 that have keyword transmembrane, when constructing multiple sequence alignments.

The dataset was derived from the Swiss-Prot database, so we make sure to remove all entries for the dataset from the two databases that we use. 


\subsection{Algorithm}

Figure 1 ilustrates the steps of TranCEP. The sequence (a) in this case has four transmembrane segments (TMS) as shown by the gray shading. The example focusses on the first TMS, and abbreviates the middle section of the sequence. Part (b) shows an MSA conserving the TMS structure constructed by TM-Coffee, where the gray shading indicates the TMS location. Part (c) shows the colour coding of the reliability index of each column as determined by TCS, and shows how gaps replace unreliable columns in the filtered MSA. Part (d) shows a 400-dimensional vector of dipeptide frequencies (the PAAC composition) from the filtered MSA.

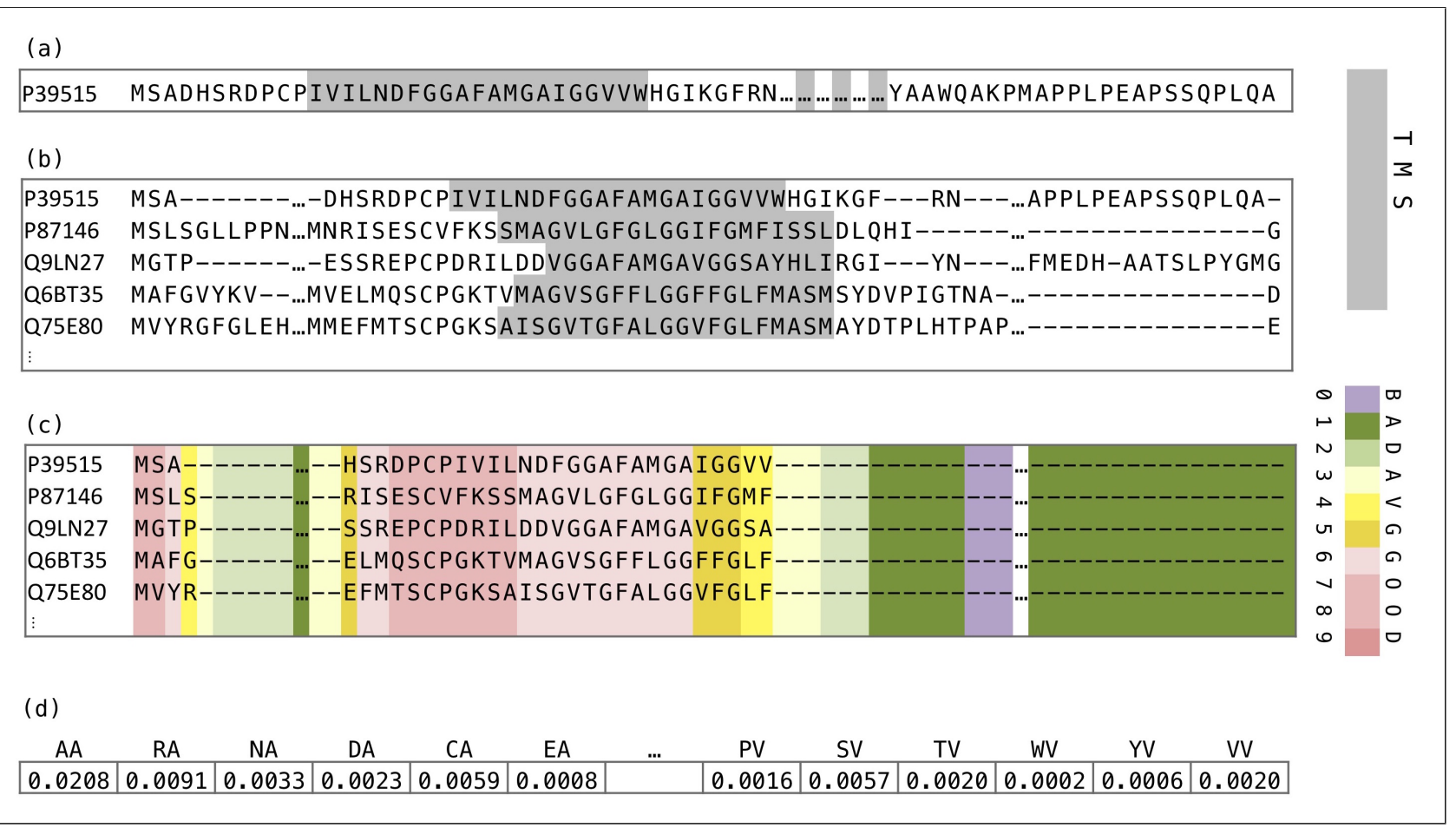

\section{Fig 1. Example of Steps of TranCEP.}

The ilustrates the steps of TranCEP. Note that we abbreviate the middle section of the sequence. Part (a) shows the sequence the four TMS in gray shading. Part (b) shows an MSA constructed by TM-Coffee. Gray shading indicates a TMS. Part (c) shows the colour coding of the reliability index of each column as determined by TCS, and shows gaps in unreliable columns in the filtered MSA. Part (d) shows a 400-dimensional PAAC vector from the filtered MSA.

The template for combining evolutionary, positional, and composition information is presented in Algorithm 1. In this work, we use TM-Coffee to compute multiple sequence alignments (MSA) that conserve the transmembrane segments, and TCS to determine a reliability index for each position (column) in the MSA. We experimented with three composition schemes: amino acid composition (AAC), pairwise amino acid composition (PAAC), and pseudo-amino acid composition (PseAAC); and the optional use of TM-Coffee (TMC) and TCS. 


\section{Algorithm 1. Template of algorithm for composition vector. function COMP_VEC(seq $s$ ) \\ // Evolutionary (E) Step, optional \\ Construct MSA from $s$ \\ // Positional (P) Step, optional \\ Determine informative positions (columns) in MSA \\ Filter uninformative posiitions from MSA \\ // Composition (C) Step, mandatory \\ return vector encoding composition of filtered MSA \\ end function}

A template for the algorithm showing the role of evolutionary $(\mathrm{E})$, positional $(\mathrm{P})$, and composition $(\mathrm{C})$ information. Note that the use of evolutionary $(\mathrm{E})$ and positional $(\mathrm{P})$ is optional; and that if positional $(\mathrm{P})$ information is used then it requires evolutionary (E) information in the form of a multiple sequence alignment (MSA). Note that if Step (E) not done, then Step (C) encodes the sequence $s$. Note that if Step (E) is done but Step (P) MSA is not done, then Step (C) encodes the MSA.

Algorithm 2 shows the composition vectors being used to build a set of classifiers; SVM classifiers in this case. Algorithm 3 shows the prediction algorithm.

\section{Algorithm 2. Build SVM classifiers.}

Require: a training set $T$ of sequences labelled with classes $C_{1}, \ldots, C_{n}$ Ensure: a set of SVM's $\operatorname{svm}(i, j)$ distinguishing class $C_{i}$ and $C_{j}$ procedure BuILD_SVMs( $T$ : set of seq; svm: set of SVM )

for all seq $s$ in $T$ do

$$
v(s) \leftarrow \mathrm{COMP}_{-} \mathrm{VEC}(s)
$$

end for

for all pair $\left(C_{i}, C_{j}\right)$ of classes do

$$
\operatorname{svm}(i, j) \leftarrow \operatorname{SVM} \text {.build }\left(\left\{v(s): s \in T \cap\left(C_{i} \cup C_{j}\right)\right\}\right)
$$

\section{end for}

end procedure

The algorithm to build a set of SVM classifiers to distinguish between each pair of classes in the training set. The actual construction of each SVM is done by an SVMM package's build function. 


\section{Algorithm 3. Prediction.}

Require: test sequence $s$

Require: a set of SVM's svm $(i, j)$ distinguishing classes $C_{i}$ and $C_{j}$

Ensure: result is predicted class $C_{p}$

function PREDICT_CLASS $(\operatorname{seq} s)$

$v \leftarrow \mathrm{COMP}_{-} \mathrm{VEC}(s)$

for all pair $\left(C_{i}, C_{j}\right)$ of classes do $c(i, j) \leftarrow \operatorname{svm}(i, j)$ applied to $v$

end for

$p \leftarrow$ mode of $c(i, j)$ for all pairs $(i, j)$

return $C_{p}$

end function

The prediction algorithm that applies each of the SVMs, and takes the class that is predicted most often by the set of SVMs.

\subsection{Encoding amino acid composition}

The properties of the amino acids at each position in the protein sequence can be encoded into vectors that summarize the overall composition of the protein. We implemented three approaches to encoding amino acid composition: $A A C, P A A C$, and PseAAC.

The Amino Acid Composition (AAC) is the normalized occurrence frequency of each amino acid. The fractions of all 20 natural amino acids are calculated as:

$$
c_{i}=\frac{F_{i}}{L} \quad i=(1,2,3, \ldots 20)
$$

where $F_{i}$ is the frequency of the $i^{t h}$ amino acid and $L$ is the length of the sequence. The AAC is represented as a vector of size 20 :

$$
A A C(P)=\left[c_{1}, c_{2}, c_{3}, \ldots, c_{20}\right]
$$

where $c_{i}$ is the composition of $i^{\text {th }}$ amino acid.

The Pair Amino Acid Composition (PAAC) is the normalized frequency of each pair of amino acids. The PAAC is calculated as

$$
d_{i, j}=\frac{F_{i, j}}{L-1} \quad i, j=(1,2,3, \ldots 20)
$$

where $F_{i, j}$ is the frequency of the $i^{\text {th }}$ and $j^{\text {th }}$ amino acids as a pair (dipeptide) and $L$ is the length of the sequence. The PAAC is represented as a vector of size 400 as follows:

$$
P A A C(P)=\left[d_{1,1}, d_{1,2}, d_{1,3}, \ldots, d_{20,20}\right]
$$

where $d_{i, j}$ is the dipeptide composition of the $i^{\text {th }}$ and $j^{\text {th }}$ amino acid.

The Pseudo-Amino Acid Composition (PseAAC) Cho01] combines the 20 components of AAC with a set of sequence order correlation factors that incorporates some biochemical properties. Given a 
protein sequence $R_{1} R_{2} R_{3} R_{4} \ldots R_{L}$ of length $L$, a set of descriptors called sequence order-correlated factors are defined as:

$$
\left\{\begin{array}{c}
\theta_{1}=\frac{1}{L-1} \sum_{i=1}^{L-1} \Theta\left(R_{i}, R_{i+1}\right) \\
\theta_{2}=\frac{1}{L-2} \sum_{i=1}^{L-2} \Theta\left(R_{i}, R_{i+2}\right) \\
\theta_{3}=\frac{1}{L-3} \sum_{i=1}^{L-3} \Theta\left(R_{i}, R_{i+3}\right) \\
\cdot \\
\cdot \\
\theta_{\lambda}=\frac{1}{L-\lambda} \sum_{i=1}^{L-\lambda} \Theta\left(R_{i}, R_{i+\lambda}\right)
\end{array}\right.
$$

The parameter $\lambda$ is chosen such that $(\lambda<L)$. A correlation function is given by:

$$
\Theta\left(R_{i}, R_{j}\right)=\frac{1}{3}\left\{\left[H_{1}\left(R_{j}\right)-H_{1}\left(R_{i}\right)\right]^{2}+\left[H_{2}\left(R_{j}\right)-H_{2}\left(R_{i}\right)\right]^{2}+\left[M\left(R_{j}\right)-M\left(R_{i}\right)\right]^{2}\right\}
$$

where $H_{1}(R)$ is the hydrophobicity value, $H_{2}(R)$ is hydrophilicity value, and $M(R)$ is side chain mass of the amino acid $R_{i}$. Those quantities are converted from their original values. For eaxmple, for hydrophobicity, $H_{1}(R)$ is derived from $H_{1}^{\circ}\left(R_{i}\right)$, as follows:

$$
H_{1}\left(R_{i}\right)=\frac{H_{1}^{\circ}\left(R_{i}\right)-\frac{1}{20} \sum_{k=1}^{20} H_{1}^{\circ}\left(R_{k}\right)}{\sqrt{\frac{\sum_{y=1}^{20}\left[H_{1}^{\circ}\left(R_{y}\right)-\frac{1}{20} \sum_{k=1}^{20} H_{1}^{\circ}\left(R_{k}\right)\right]^{2}}{20}}}
$$

The original hydrophobicity value $H_{1}^{\circ}\left(R_{i}\right)$ is taken from Tanford [Tan62]. The original hydrophilicity value $H_{2}^{\circ}\left(R_{i}\right)$ for the amino acid $R_{i}$ is taken from Hopp and Woods [HW81]. The mass $M^{\circ}\left(R_{i}\right)$ of the $R_{i}$ amino acid side chain can be obtained from any biochemistry text book.

PseAAC is represented as vector of size $(20+\lambda)$ as follows:

$$
\operatorname{Pse} A A C(P)=\left[s_{1}, \ldots, s_{20}, s_{21}, \ldots, s_{20+\lambda}\right]
$$

where $s_{i}$ is the pseudo-amino acid composition

$$
s_{i}=\left\{\begin{array}{lc}
\frac{f_{i}}{\sum_{r=1}^{20} f_{r}+\omega \sum_{j=1}^{\lambda} \theta_{j}} & 1 \leq i \leq 20 \\
\frac{\omega \theta_{i-20}}{\sum_{r=1}^{20} f_{r}+\omega \sum_{j=1}^{\lambda} \theta_{j}} & 20<i \leq 20+\lambda
\end{array}\right.
$$


where $f_{i}$ is the normalized occurrence frequency of the of the $i t h$ amino acid in the protein sequence, $\theta_{j}$ is the $j^{\text {th }}$ sequence order-correlated factor calculated from Equation 5 , and $\omega$ is a weight factor for the sequence order effect. The weight factor $\omega$ puts weight on the additional PseAAC components with respect to the conventional AAC components. The user can select any value from 0.05 to 0.7 for the weight factor. The default value given by Chou Cho01] is .05.

\subsection{Multiple sequence alignment}

We adopted the $M S A-A A C$ approach [SCH10] that combines amino acid composition with the evolutionary information available from a multiple sequence alignment (MSA). This is done by first retrieving homologous sequences of each protein sequence in the dataset, then building a MSA for the corresponding protein, then finally taking the counts for computing the composition information using all the residues in the MSA.

In [SCH10], they only used the $A A C$ encoding, while we also applied the approach to the $P A A C$ and PseAAC encodings. Another difference is that we adopted TM-COFFEE [CDTTN12] (Version11.00.8cbe486) to compute alignments, rather than use ClustalW [THG94] as done in [SCH10], as we feel it is important to align the transmembrane segments well.

Other differences include searching the Swiss-Prot $\left[\mathrm{BBA}^{+} 03\right]$ database and retrieving a maximum of 120 homologous sequences instead of searching the non-redundant database $n r$ and retrieving 1000 sequences. We sought to make the computational time more manageable because TM-COFFEE algorithm requires more memory usage and execution time.

Furthermore, all exact hits of the test sequences were removed from the Swiss-Prot and UniRef50-TM databases to maintain independence between the MSA and the test data.

Our alignment command was the following: :

$$
\begin{aligned}
& \text { t_coffee mysequences.fasta -mode psicoffee } \\
& \text { - protein_db uniref50-TM । } \\
& \text {-template_file PSITM }
\end{aligned}
$$

where mysequences. fasta contains the 120 similar sequences retrieved by BLAST.

\subsection{Positional information}

In order to focus the information on those positions in the protein that determine specificity, we need a method to determine those positions, and then to filter our information. The information is filtered by setting the entries for all other positions to null, that is, the symbol '- ' so that it is ignored when gathering counts for the amino acid composition information.

For determining the positions, we applied the transitive consistency score (TCS) algorithm [CDTN14] to the alignment. The TCS is a scoring scheme that uses a consistency transformation to assign a reliability index to every pair of aligned residues, to each individual residue in the alignment, to each column, and to the overall alignment. This scoring scheme is shown to be highly informative with respect to structural accuracy prediction based on benchmarking databases. The reliably index 
ranges from 0 to 9 , where 0 is extremely uncertain and 9 is very reliable. Columns with a reliability index of below 4 were removed using the following command:

$$
\begin{gathered}
\text { t_coffee - infile myMSA.aln - evaluate } \\
\text { - output tcs_column_filter } 4 \text {.fasta }
\end{gathered}
$$

where myMSA.aln is the MSA file, tcs_column_filter4.fasta is the filtered file in FASTA format.

\subsection{Training}

Following TrSSP [MCZ14], we use a multi-class SVM with RBF kernel as implemented by the $\mathrm{R}$ e1071 library version 1.6-8 using a one-against-one approach in which $21=(7 \times 6) / 2$ binary classifiers are trained. The predicted class is determined through a voting scheme where all the binary classifiers are applied and the class that gets highest number of votes is the result. We also optimized both cost and gamma parameters of RBF kernel by performing grid search using tune function in the library (range of cost: 2 - 16, gamma: 2-e-5 - 1).

\subsection{Methods}

We implemented three approaches to encoding amino acid composition: $A A C, P A A C$ as done by TrSSP [MCZ14], and PseAAC. These were followed by training using SVM to form the prediction methods AAC, PAAC, and PseAAC respectively.

By combining the amino acid composition and the evolutionary information obtained using TMCoffee, followed by SVM, we implemented the prediction methods: TMC-AAC, TMC-PAAC, and TMC-PseAAC respectively.

Filtering was incorporated by applying TCS after TM-Coffee, then computing the amino acid composition vectors, and applying SVM. to implement the prediction methods: TMC-TCS-AAC, TMC-TCS-PAAC, and TMC-TCS-PseAAC respectively.

The method TranCEP is TMC-TCS-PAAC, as it gave the best performance during crossvalidation.

\subsection{Performance measurement}

Four statistical measures were considered to measure the performance:

sensitivity which is the proportion of positives that are correctly identified:

$$
\text { Sensitivity }=\frac{T P}{T P+F N}
$$

specificity which is the proportion of negatives that are correctly identified:

$$
\text { Specificity }=\frac{T N}{T N+F P}
$$


accuracy which is proportion of correct predictions made amongst all the predictions:

$$
\text { Accuracy }=\frac{T P+T N}{T P+F N+T N+F P}
$$

Matthews correlation coefficient (MCC) which is a single measure taking into account true and false positives and negatives:

$$
M C C=\frac{(T P \times T N-F P \times F N)}{\sqrt{(T P+F P) \times(T P+F N) \times(T N+F P) \times(T N+F N)}}
$$

where $T P$ is the number of true positives, $T N$ is the number of true negatives, $F P$ is the number of false positives, and $F N$ is the number of false negatives.

We use the MCC because it is less influenced by imbalanced data and is arguably the best single assessment metric in this case [Din11, WP03, BDA13. The MCC value ranges from 1 to -1 , where 1 indicates a perfect prediction; 0 represent no better than random; and -1 implies total disagreement between prediction and observation. A high MCC value means the predictor has high accuracy on both positive and negative classes, and also low misprediction on both.

In the multi-class case with $K$ classes, the MCC is calculated in terms of the confusion matrix $C$ of dimension $K \times K$ Gor04]:

$$
\mathrm{MCC}=\frac{\sum_{k} \sum_{l} \sum_{m} C_{k k} C_{l m}-C_{k l} C_{m k}}{\sqrt{\sum_{k}\left(\sum_{l} C_{k l}\right)\left(\sum_{k^{\prime} \mid k^{\prime} \neq k} \sum_{l^{\prime}} C_{k^{\prime} l^{\prime}}\right)} \sqrt{\sum_{k}\left(\sum_{l} C_{l k}\right)\left(\sum_{k^{\prime} \mid k^{\prime} \neq k} \sum_{l^{\prime}} C_{l^{\prime} k^{\prime}}\right)}}
$$

\subsection{Experiments}

Performance of each method was determined using both a five-fold cross-validation where the training dataset is randomly partitioned into five equal sized sets. A single set is kept as the validation data and the remaining four sets are used to train the SVM model. This model is then tested using the validation set. The cross-validation process is repeated four times where each of the sets is used once as the validation data. The performance of each model is averaged to produce a single estimation and the variation of performances is captured by computing the stranded deviation.

The performance of TranCEP on the test dataset was compared to the performance of TrSSP as reported in their paper [MCZ14].

\section{Results and discussion}

\subsection{Cross-Validation}

The performance of the nine methods were evaluated using five-fold cross-validation. Table 2 presents the overall accuracy and MCC of SVM models for the nine methods, sorted from the best to worst MCC. All SVM models that utilized evolutionary data notably performed better 


\begin{tabular}{|c|c|c|}
\hline Method & Accuracy & MCC \\
\hline TMC-TCS-PAAC & $69.23 \pm 0.061$ & $0.63 \pm 0.122$ \\
\hline TMC-TCS-AAC & $65.13 \pm 0.032$ & $0.58 \pm 0.125$ \\
\hline TMC-PAAC & $63.33 \pm 0.044$ & $0.51 \pm 0.129$ \\
\hline TMC-AAC & $63.08 \pm 0.036$ & $0.50 \pm 0.092$ \\
\hline TMC-TCS-PseAAC & $63.30 \pm 0.055$ & $0.49 \pm 0.129$ \\
\hline TMC-PseAAC & $61.79 \pm 0.029$ & $0.49 \pm 0.131$ \\
\hline PseAAC & $47.69 \pm 0.024$ & $0.27 \pm 0.104$ \\
\hline PAAC & $45.89 \pm 0.031$ & $0.25 \pm 0.101$ \\
\hline AAC & $45.38 \pm 0.033$ & $0.22 \pm 0.099$ \\
\hline
\end{tabular}

Table 2. Overall cross-validation performance of the methods. For each method, the table presents accuracy and MCC as $m \pm d$, where $m$ is the mean and $d$ is the standard deviation across the five runs of the cross validation.

\begin{tabular}{|l||c|c|c|c|}
\hline Class & Specificity & Sensitivity & Accuracy & MCC \\
\hline Amino acid & $98.82 \pm 0.004$ & $80.84 \pm 0.054$ & $96.06 \pm 0.017$ & $0.82 \pm 0.042$ \\
\hline Anion & $99.30 \pm 0.007$ & $47.89 \pm 0.199$ & $93.33 \pm 0.014$ & $0.61 \pm 0.119$ \\
\hline Cation & $86.66 \pm 0.022$ & $79.70 \pm 0.043$ & $81.50 \pm 0.039$ & $0.62 \pm 0.081$ \\
\hline Electron & $96.58 \pm 0.030$ & $63.13 \pm 0.118$ & $92.01 \pm 0.019$ & $0.58 \pm 0.098$ \\
\hline Protein & $97.21 \pm 0.018$ & $52.00 \pm 0.087$ & $90.93 \pm 0.031$ & $0.46 \pm 0.036$ \\
\hline Sugar & $98.19 \pm 0.019$ & $70.51 \pm 0.105$ & $94.39 \pm 0.021$ & $0.52 \pm 0.192$ \\
\hline Other & $83.02 \pm 0.035$ & $67.23 \pm 0.198$ & $76.43 \pm 0.068$ & $0.69 \pm 0.178$ \\
\hline Overall & & $69.23 \pm 0.061$ & $0.63 \pm 0.122$ \\
\hline
\end{tabular}

Table 3. Cross validation performance for TMC-TCS-PAAC. The table presents cross validation results for TranCEP, which is TCSTMC-PAAC. For each substrate class, the table presents specificity, sensitivity, accuracy and MCC as $m \pm d$, where $m$ is the mean and $d$ is the standard deviation across the five runs of the cross validation.

than the SVM models that did not. In addition, the top two models, TMC-TCS-PAAC and TMC-TCS-AAC, integrated positional and evolutionary information. The highest MCC was obtained by TMC-TCS-PAAC, which is the method chosen for our predictor TranCEP.

The performances of different SVM models were evaluated using five-fold cross-validation. The highest MCC was obtained by TMC-TCS-PAAC which is the method chosen for our predictor TranCEP. This method incorporates the use of PAAC with evolutionary data in the form of MSA with positional information, in which columns that have a reliability below 4 are filtered out. We found that the performance peaked using this threshold and started to decline when filtering columns with a higher reliability index. The TMC-TCS-AAC method yielded an overall MCC of .63. The detailed performance is presented in Table 3 , MCC was 0.82, 0.61, 0.62, 0.58, 0.46, 0.52, and 0.69 for amino acid, anion, cation, electron, protein/mRNA, sugar, and other, respectively.

Table 4 presents the confusion matrix for TranCEPwhen run on the independent test set. Most of the confusion occurs between a substrate class and the class other. 
bioRxiv preprint doi: https://doi org/10.1101/293159; this version posted April 2, 2018. The copyright holder for this preprint (which was not certified by peer review) is the author/funder, who has granted bioRxiv a license to display the preprint in perpetuity. It is made available under aCC-BY-NC-ND 4.0 International license.

\begin{tabular}{|l||c|c|c|c|c|c|c||c|}
\hline Actual $\backslash$ Predicted & Amino acid & Anion & Cation & Electron & Protein & Sugar & Other & Total \\
\hline Amino acid & 9 & 0 & 2 & 0 & 0 & 0 & 4 & 15 \\
\hline Anion & 1 & 7 & 1 & 0 & 0 & 0 & 3 & 12 \\
\hline Cation & 0 & 0 & 34 & 0 & 1 & 0 & 1 & 36 \\
\hline Electron & 0 & 0 & 1 & 8 & 0 & 0 & 1 & 10 \\
\hline Protein & 0 & 0 & 3 & 0 & 10 & 0 & 2 & 15 \\
\hline Sugar & 0 & 1 & 0 & 0 & 0 & 8 & 3 & 12 \\
\hline Other & 1 & 3 & 2 & 0 & 0 & 1 & 13 & 20 \\
\hline
\end{tabular}

Table 4. Confusion matrix for TranCEP.

The table presents the number of proteins in an actual substrate class that are predicted by TranCEP to fall in each substrate class. The independent test set is used.

\subsection{Comparison}

\begin{tabular}{|c|c|c|c|c|c|c|c|c|}
\hline \multirow[t]{2}{*}{ Class } & \multicolumn{2}{|c|}{ Specificity } & \multicolumn{2}{|c|}{ Sensitivity } & \multicolumn{2}{|c|}{ Accuracy } & \multicolumn{2}{|c|}{ MCC } \\
\hline & TrSSP & TranCEP & TrSSP & TranCEP & TrSSP & TranCEP & TrSSP & TranCEP \\
\hline Amino acid & 82.42 & 98.10 & 93.33 & 60.00 & 83.33 & 91.75 & 0.49 & 0.66 \\
\hline Anion & 69.05 & 96.30 & 75.00 & 58.33 & 69.44 & 90.82 & 0.23 & 0.56 \\
\hline Cation & 74.31 & 89.29 & 75.00 & 94.44 & 74.44 & 89.00 & 0.41 & 0.78 \\
\hline Electron & 91.78 & 99.05 & 80.00 & 80.00 & 91.11 & 97.80 & 0.50 & 0.88 \\
\hline Protein & 82.42 & 99.07 & 93.33 & 66.67 & 83.33 & 93.68 & 0.49 & 0.75 \\
\hline Sugar & 76.79 & 99.07 & 91.67 & 66.67 & 77.78 & 94.68 & 0.38 & 0.74 \\
\hline Other & 73.13 & 86.00 & 60.00 & 65.00 & 71.67 & 80.91 & 0.23 & 0.44 \\
\hline Overall & & & & & 78.88 & 74.17 & 0.41 & 0.69 \\
\hline
\end{tabular}

Table 5. Comparing TranCEP and TrSSP.

The table presents our data for TranCEP built with the training set and run on the test set. The corresponding results for TrSSP are taken from their original paper. The table shows specificity, sensitivity, accuracy and MCC for each of the seven substrate types; and the average accuracy and MCC. We calculated accuracy as proportion of correct predictions divided by the total number of predictions, and MCC from the confusion matrix as in Equation 14. The TrSSP results were calculated as the average across the seven classes; if we adopt the same method the average accuracy is $91.27 \%$ and the average MCC is 0.69 .

Table 5 compares the performance of TranCEP to TrSSP [MCZ14 on the independent test set. For all substrate classes, TranCEP scored higher in accuracy, specificity, and MCC. However, for the true positive rate as measured by sensitivity, TrSSP performed better on the cation and other classes, and TranCEP either matched or outperformed TrSSP on the other five classes. Overall, TranCEP obtained MCC of 0.69 which is $68 \%$ higher than that of TrSSP.

\subsection{Impact of Factors}

Table 6 presents the impact of each factor - evolutionary information and positional information - on the overall MCC. The use of evolutionary information in form of MSA on the composition encodings AAC, PAAC, and PseAAC has improved the MCC by an average of $104 \%$ with the highest improvement being on AAC by $127 \%$. The further use of positional information by filtering out the unreliable columns from the MSA has boosted the MCC of the composition encodings by an average of $132 \%$. The impact of positional information over that already achieved by evolutionary 


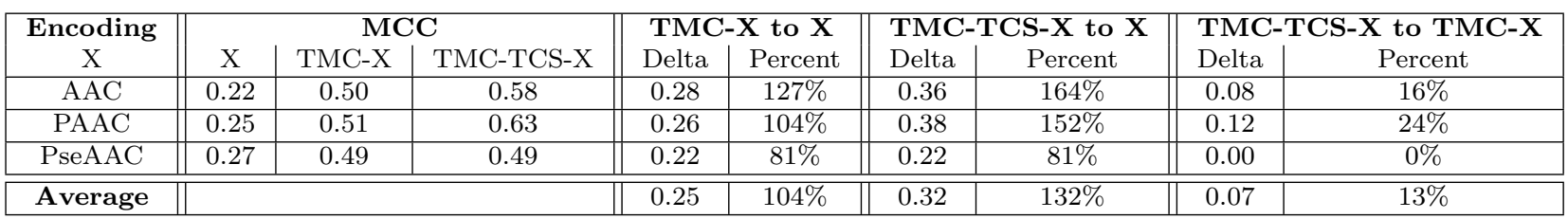

Table 6. Impact of factors on performance.

The table notes the difference in MCC and the percentage improvement in MCC of the crossvalidation performance for the introduction of evolutionary information using TM-Coffee, and positional information using TCS.

information was an average of 14\%. The highest impact was on TMC-PAAC, where the MCC improved by $24 \%$ in TMC-TCS-PAAC.

\begin{tabular}{|c|c|c|c|c|c|c|c|c|c|c|c|}
\hline \multirow[t]{2}{*}{ Class } & \multirow[t]{2}{*}{ SeqLth } & \multirow[t]{2}{*}{ TMS } & \multirow[t]{2}{*}{ TMSLth } & \multicolumn{2}{|c|}{ Positions } & \multicolumn{3}{|c|}{ TMS } & \multicolumn{3}{|c|}{ non-TMS } \\
\hline & & & & Num & \%Seq & Num & $\begin{array}{c}\text { Interior } \\
\text { Num }\end{array}$ & $\begin{array}{c}\text { Exterior } \\
\text { Num }\end{array}$ & Num & $\begin{array}{l}\text { Close } \\
\text { Num }\end{array}$ & $\begin{array}{c}\text { Far } \\
\text { Num }\end{array}$ \\
\hline Amino acid & 535 & 11 & 218 & 310 & 58.83 & 165 & 63 & 103 & 146 & 82 & 64 \\
\hline Anion & 632 & 10 & 209 & 441 & 71.64 & 179 & 67 & 112 & 267 & 100 & 167 \\
\hline Cation & 644 & 8 & 164 & 398 & 63.31 & 135 & 51 & 84 & 280 & 78 & 202 \\
\hline Electron & 352 & 5 & 103 & 262 & 76.62 & 88 & 32 & 55 & 243 & 49 & 194 \\
\hline Protein & 592 & 3 & 57 & 284 & 53.79 & 35 & 13 & 22 & 283 & 26 & 257 \\
\hline Sugar & 467 & 11 & 222 & 312 & 66.90 & 188 & 72 & 116 & 131 & 75 & 56 \\
\hline Other & 580 & 9 & 180 & 368 & 65.27 & 136 & 52 & 85 & 253 & 74 & 179 \\
\hline
\end{tabular}

Table 7. Positional information.

The table present information on the sites retained by the TCS filtering step. For each class of substrates in the dataset, the table presents the average sequence length (SeqLth), the average number of TMS regions (TMS), and the average total number of residues in the TMS regions (TMSLth). It also presents the average of the number of positions retained by the filtering (Positions:Num), and that number as a percentage of the total sequence length (Positions:\%Seq). It notes the total number of sites that occur in the TMS regions (TMS:Num), and the non-TMS regions (non-TMS:Num). For the TMS regions, it presents the average number of sites that occur in the interior central one-third of the TMS regions (TMS:Interior:Num), and in the remaining exterior regions outside the central one-third of the TMS regions (TMS:Exterior:Num). For the non-TMS regions, it presents the average number of sites that occur close to the TMS regions (within 10 positions of the TMS) (non-TMS:Close:Num), and the remaining sites far from the TMS regions (non-TMS:Far:Num).

Table 7 presents a breakdown on where the informative positions, as determined by TCS, are located with respect to the tramsmembrane segments (TMS). The protein sequence positions were divided into those in the TMS and those not in the TMS. Those in the TMS were divided into the interior one-third positions, and the remaining exterior positions in the TNS. The non-TMS positions were divided into those near a TMS, that is, within 10 positions), and the remaining positions far from a TMS. The location of the TMS was predicted using HMMTOP [TS01] to predict the $\alpha$-helical TMS and PRED-TMBB2 [TEB16] for $\beta$-barrel TMS. Overall the positional information filtered out an average of $(35 \% \pm 7 \%)$ of the sequence. We found that amino acid, anion, cation, sugar, and other, substrates have significantly more important positions in the TMS compared with non-TMS and significantly more important positions close to TMS compared with positions far from TMS (Student's t-test, $\mathrm{P}<0.0001$ ). In contrast, in protein $/ m R N A$ and electron substrates, the difference was not significant. This could be because these two classes have fewer TMSs, and TCS did not capture their conservation. 


\section{Conclusion}

We have developed a novel method TranCEP for de novo prediction of substrates for membrane transporter proteins that combines information based on amino acid composition, evolutionary information, and positional information. TranCEP incorporates first, the use of evolutionary information taking 120 similar sequences and constructing a multiple sequence alignment using TM-Coffee, second, the use of positional information by filtering to reliable positions as determined by TCS, and third, the use of pair amino acid composition. TranCEP achieved an overall MCC of 0.69 , which is a $68 \%$ improvement over the state-of-the-art method TrSSP that uses the primary sequence alone. In addition, we evaluated the impact on performance of each factor: incorporating evolutionary information and filtering the unreliable positions. We observed that using amino acid composition alone does not obtain strong performance. The enhanced performance came from incorporating evolutionary and positional information. We learned that certain positions in the alignment have greater significance, and identifying them helped to boost the performance.

\section{References}

[AB17] Faizah Aplop and Greg Butler. TransATH: Transporter prediction via annotation transfer by homology. ARPN Journal of Engineering and Applied Sciences, 12(2), January 2017.

[Apl16] Faizah Aplop. Computational Approaches To Improving The Reconstruction Of Metabolic Pathway. PhD thesis, Concordia University, 2016.

$\left[\mathrm{BBA}^{+} 03\right]$ Brigitte Boeckmann, Amos Bairoch, Rolf Apweiler, Marie-Claude Blatter, Anne Estreicher, Elisabeth Gasteiger, Maria J Martin, Karine Michoud, Claire O’Donovan, Isabelle Phan, et al. The SWISS-PROT protein knowledgebase and its supplement TrEMBL in 2003. Nucleic Acids Research, 31(1):365-370, 2003.

[BDA13] Mohamed Bekkar, Hassiba Kheliouane Djemaa, and Taklit Akrouf Alitouche. Evaluation measures for models assessment over imbalanced data sets. Journal of Information Engineering and Applications, 3(10), 2013.

[BGA $\left.{ }^{+} 17\right]$ Basharat Bhat, Nazir A Ganai, Syed Mudasir Andrabi, Riaz A Shah, and Ashutosh Singh. TM-Aligner: Multiple sequence alignment tool for transmembrane proteins with reduced time and improved accuracy. Scientific reports, 7(1):12543, 2017.

[BH13] Ahmad Barghash and Volkhard Helms. Transferring functional annotations of membrane transporters on the basis of sequence similarity and sequence motifs. $B M C$ Bioinformatics, 14(1):343, 2013.

[BRK17] Ahmad Hassan Butt, Nouman Rasool, and Yaser Daanial Khan. A treatise to computational approaches towards prediction of membrane protein and its subtypes. The Journal of Membrane Biology, 250(1):55-76, 2017. 
[BSJ03] W Busch and MH Saier Jr. The IUBMB-endorsed transporter classification system. Methods in Molecular Biology, 227:21, 2003.

[Bue15] Lukas Buehler. The Structure of Membrane Proteins. In Cell Membranes, chapter 3. Garland Science, 2015.

[CC14] Abhijit Chakraborty and Saikat Chakrabarti. A survey on prediction of specificitydetermining sites in proteins. Briefings in Bioinformatics, page bbt092, 2014.

[CDTN14] Jia-Ming Chang, Paolo Di Tommaso, and Cedric Notredame. TCS: a new multiple sequence alignment reliability measure to estimate alignment accuracy and improve phylogenetic tree reconstruction. Molecular Biology and Evolution, pages 1625-1637, 2014.

[CDTTN12] Jia-Ming Chang, Paolo Di Tommaso, Jean-François Taly, and Cedric Notredame. Accurate multiple sequence alignment of transmembrane proteins with PSI-Coffee. BMC Bioinformatics, 13(Suppl 4):S1, 2012.

[Cho01] Kuo-Chen Chou. Prediction of protein cellular attributes using pseudo-amino acid composition. Proteins: Structure, Function, and Bioinformatics, 43(3):246-255, 2001.

[COLG11] ShuAn Chen, YuYen Ou, TzongYi Lee, and M Michael Gromiha. Prediction of transporter targets using efficient RBF networks with PSSM profiles and biochemical properties. Bioinformatics, 27(15):2062-2067, 2011.

[DGV ${ }^{+}$17] Oscar Dias, Daniel Gomes, Paulo Vilaça, João Cardoso, Miguel Rocha, Eugénio C Ferreira, and Isabel Rocha. Genome-wide semi-automated annotation of transporter systems. IEEE/ACM Transactions on Computational Biology and Bioinformatics, $14(2): 443-456,2017$.

[Din11] Zejin Ding. Diversified ensemble classifiers for highly imbalanced data learning and their application in bioinformatics. PhD thesis, Georgia State University, 2011.

[DRFR15] Oscar Dias, Miguel Rocha, Eugénio C Ferreira, and Isabel Rocha. Reconstructing genome-scale metabolic models with merlin. Nucleic Acids Research, page gkv294, 2015.

[FBS $\left.{ }^{+} 14\right] \quad$ Alexander Farwick, Stefan Bruder, Virginia Schadeweg, Mislav Oreb, and Eckhard Boles. Engineering of yeast hexose transporters to transport D-xylose without inhibition by D-glucose. Proceedings of the National Academy of Sciences, 111(14):5159-5164, 2014.

[FTC ${ }^{+}$16] Evan W Floden, Paolo D Tommaso, Maria Chatzou, Cedrik Magis, Cedric Notredame, and Jia-Ming Chang. PSI/TM-Coffee: a web server for fast and accurate multiple sequence alignments of regular and transmembrane proteins using homology extension on reduced databases. Nucleic Acids Research, 44(W1):W339-W343, 2016.

[GO14] MM Gromiha and YY Ou. Bioinformatics approaches for functional annotation of membrane proteins. Briefings in Bioinformatics, 15(2):155-168, 2014. 
[Gor04] Jan Gorodkin. Comparing two K-category assignments by a K-category correlation coefficient. Computational Biology and Chemistry, 28(5):367-374, 2004.

[GY08] M Michael Gromiha and Yukimitsu Yabuki. Functional discrimination of membrane proteins using machine learning techniques. BMC Bioinformatics, 9(1):135, 2008.

[HW81] Thomas P Hopp and Kenneth R Woods. Prediction of protein antigenic determinants from amino acid sequences. Proceedings of the National Academy of Sciences, 78(6):3824$3828,1981$.

[KST13] Dániel Kozma, István Simon, and Gábor E Tusnády. PDBTM: Protein data bank of transmembrane proteins after 8 years. Nucleic Acids Research, 41(D1):D524-D529, 2013.

[LBUZ09] Haiquan Li, Vagner A Benedito, Michael K Udvardi, and Patrick Xuechun Zhao. TransportTP: A two-phase classification approach for membrane transporter prediction and characterization. BMC Bioinformatics, 10(418):1-13, 2009.

[LDNS12] Nicolas Loira, Thierry Dulermo, Jean-Marc Nicaud, and David J Sherman. A genomescale metabolic model of the lipid-accumulating yeast Yarrowia lipolytica. BMC Systems Biology, 6(1):35, 2012.

[LDZ08] Haiquan Li, Xinbin Dai, and Xuechun Zhao. A nearest neighbor approach for automated transporter prediction and categorization from protein sequences. Bioinformatics, 24(9):1129-1136, 2008.

$\left[\mathrm{LHC}^{+}\right.$06] HH Lin, LY Han, CZ Cai, ZL Ji, and YZ Chen. Prediction of transporter family from protein sequence by support vector machine approach. Proteins: Structure, Function, and Bioinformatics, 62(1):218-231, 2006.

[LLX+16] Liqi Li, Jinhui Li, Weidong Xiao, Yongsheng Li, Yufang Qin, Shiwen Zhou, and Hua Yang. Prediction the substrate specificities of membrane transport proteins based on support vector machine and hybrid features. IEEE/ACM Transactions on Computational Biology and Bioinformatics, 13(5):947-953, 2016.

[Loi12] Nicolas Loira. Scaffold-based Reconstruction Method for Genome-Scale Metabolic Models. PhD thesis, Université Sciences et Technologies-Bordeaux I, 2012.

[LPK08] Thomas J. Lee, Ian Paulsen, and Peter Karp. Annotation-based inference of transporter function. Critical Reviews in Biochemistry and Molecular Biology, 24:i259-i267, 2008.

$\left[\mathrm{LVY}^{+} 15\right] \quad$ Yi-Fan Liou, Tamara Vasylenko, Chia-Lun Yeh, Wei-Chun Lin, Shih-Hsiang Chiu, Phasit Charoenkwan, Li-Sun Shu, Shinn-Ying Ho, and Hui-Ling Huang. SCMMTP: identifying and characterizing membrane transport proteins using propensity scores of dipeptides. BMC Genomics, 16(12):S6, 2015.

[LZS15] Nicolas Loira, Anna Zhukova, and David James Sherman. Pantograph: A templatebased method for genome-scale metabolic model reconstruction. Journal of Bioinformatics and Computational Biology, 13(02):1550006, 2015. 
[MCZ14] Nitish K. Mishra, Junil Chang, and Patrick X. Zhao. Prediction of membrane transport proteins and their substrate specificities using primary sequence information. PLoS One, 9(6):1-14, 2014.

[OCG10] Yu-Yen Ou, Shu-An Chen, and M Michael Gromiha. Classification of transporters using efficient radial basis function networks with position-specific scoring matrices and biochemical properties. Proteins: Structure, Function, and Bioinformatics, 78(7):17891797, 2010.

[PFH08] Walter Pirovano, K Anton Feenstra, and Jaap Heringa. PRALINE ${ }^{\mathrm{TM}}$ : a strategy for improved multiple alignment of transmembrane proteins. Bioinformatics, 24(4):492-497, 2008.

[PVL $\left.{ }^{+} 14\right] \quad$ Philipp Paparoditis, Åke Västermark, Andrew J Le, John A Fuerst, and Milton H Saier. Bioinformatic analyses of integral membrane transport proteins encoded within the genome of the planctomycetes species, Rhodopirellula baltica. Biochimica et Biophysica Acta (BBA)-Biomembranes, 1838(1):193-215, 2014.

[RCP07] Qinghu Ren, Kaixi Chen, and Ian T. Paulsen. TransportDB: a comprehensive database resource for cytoplasmic membrane transport systems and outer membrane channels. Nucleic Acids Research, 35:D274-D279, 2007.

[RKP04] Qinghu Ren, Katherine H Kang, and Ian T Paulsen. TransportDB: a relational database of cellular membrane transport systems. Nucleic Acids Research, 32(suppl 1):D284-D288, 2004.

[RS12] Vamsee S Reddy and Milton H Saier. BioV Suite - a collection of programs for the study of transport protein evolution. FEBS Journal, 279(11):2036-2046, 2012.

[SAJT14] Swagatika Sahoo, Maike Kathrin Aurich, Jon Johannes Jonsson, and Ines Thiele. Membrane transporters in a human genome-scale metabolic knowledgebase and their implications for disease. Frontiers in physiology, 5:91, 2014.

[SCH10] Nadine S Schaadt, Jan Christoph, and Volkhard Helms. Classifying substrate specificities of membrane transporters from Arabidopsis thaliana. Journal of Chemical Information and Modeling, 50(10):1899-1905, 2010.

[SH12] NS Schaadt and V Helms. Functional classification of membrane transporters and channels based on filtered TM/non-TM amino acid composition. Biopolymers, 97(7):558$567,2012$.

[SJRT ${ }^{+}$16] Milton H Saier Jr, Vamsee S Reddy, Brian V Tsu, Muhammad Saad Ahmed, Chun $\mathrm{Li}$, and Gabriel Moreno-Hagelsieb. The transporter classification database (TCDB): recent advances. Nucleic Acids Research, 44(D1):D372-D379, 2016.

[SJTB06] Milton H Saier Jr, Can V Tran, and Ravi D Barabote. TCDB: the Transporter Classification Database for membrane transport protein analyses and information. Nucleic Acids Research, 34(suppl_1):D181-D186, 2006. 
[Tan62] Charles Tanford. Contribution of hydrophobic interactions to the stability of the globular conformation of proteins. Journal of the American Chemical Society, 84(22):4240-4247, 1962.

[TEB16] Konstantinos D Tsirigos, Arne Elofsson, and Pantelis G Bagos. PRED-TMBB2: improved topology prediction and detection of beta-barrel outer membrane proteins. Bioinformatics, 32(17):i665-i671, 2016.

[THG94] Julie D Thompson, Desmond G Higgins, and Toby J Gibson. CLUSTAL W: improving the sensitivity of progressive multiple sequence alignment through sequence weighting, position-specific gap penalties and weight matrix choice. Nucleic Acids Research, 22(22):4673-4680, 1994.

[TP10] Ines Thiele and Bernhard $\varnothing$ Palsson. A protocol for generating a high-quality genomescale metabolic reconstruction. Nature protocols, 5(1):93-121, 2010.

[TS01] Gabor E Tusnady and Istvan Simon. The HMMTOP transmembrane topology prediction server. Bioinformatics, 17(9):849-850, 2001.

[TWNB12] Elin Teppa, Angela D Wilkins, Morten Nielsen, and Cristina Marino Buslje. Disentangling evolutionary signals: conservation, specificity determining positions and coevolution. implication for catalytic residue prediction. BMC Bioinformatics, 13(1):235, 2012.

[WP03] Gary M Weiss and Foster Provost. Learning when training data are costly: The effect of class distribution on tree induction. Journal of Artificial Intelligence Research, 19:315-354, 2003. 